\title{
Um Estudo sobre a Variabilidade de Aspectos Dinâmicos no Ecossistema de Software Educacional SOLAR
}

\author{
Emanuel Ferreira Coutinho $^{1,2}$, Carla Ilane Moreira Bezerra ${ }^{1,2}$ \\ ${ }^{1}$ Programa de Pós-Graduação em Computação (PCOMP) \\ ${ }^{2}$ Universidade Federal do Ceará (UFC) - Quixadá, Brazil \\ emanuel.coutinhodufc.br, carlailanedufc.br
}

\begin{abstract}
A Software Ecosystem (SECO) refers to a collection of software products with some degree of symbiotic relationship. SOLAR is a Virtual Learning Environment (VLE) that allows courses publication and interaction with them among its various users. In this context, SOLAR SECO arises, where several evolution and maintenance situations are part of their daily. The objective of this work is to discuss software modeling dynamic aspects of SOLAR e-learning SECO. As an example, we discussed dynamic variability aspects of the SOLAR $V L E$ features model, specifically the discussion forum functionality, one of the most used services within the SOLAR SECO.
\end{abstract}

Resumo. Um Ecossistema de Software (ECOS) refere-se a uma coleção de produtos de software com algum determinado grau de relacionamento simbiótico. O SOLAR é um Ambiente Virtual de Aprendizagem (AVA) que possibilita a publicação de cursos e interação com os mesmos entre seus diversos usuários. Nesse contexto, surge o ECOS SOLAR, onde diversas situações de evolução e manutenção fazem parte de seu cotidiano. O objetivo deste trabalho é discutir a variabilidade dinâmica da modelagem de software do ECOS de e-learning SOLAR. Como exemplo, discutiu-se aspectos de variabilidade dinâmica do modelo de features da funcionalidade fórum de discussão do AVA SOLAR, um dos serviços mais utilizados dentro do ECOS SOLAR.

\section{Introdução}

De acordo com a Norma Internacional ISO/IEC/ IEEE 42010, um sistema intensivo de software é um sistema no qual o software essencialmente influencia o projeto, construção, implantação e evolução do sistema como um todo para abranger aplicações individuais, subsistemas, sistemas de sistemas, linhas de produtos, famílias de produtos, empresas inteiras e outras agregações de interesse [ISO 2011]. A complexidade do software e a complexidade dos sistemas dependentes de software cresceram a um ritmo intenso [Oquendo 2016]. Em particular, sistemas intensivos de software foram rapidamente evoluídos de sistemas independentes no passado, para fazer parte de sistemas em rede no presente, e para se tornarem sistemas de sistemas no futuro próximo.

Tradicionalmente, um Ecossistema de Software (ECOS) refere-se a uma coleção de produtos de software com algum determinado grau de relacionamento simbiótico [Messerschmitt and Szyperski 2003]. Um ECOS também pode consistir em um conjunto 
de atores agindo como uma unidade que interage com um mercado distribuído entre software e serviços, juntamente com as relações entre estas entidades [Jansen et al. 2009]. Tais relações são frequentemente apoiadas por uma plataforma tecnológica ou por um mercado comum e realizadas pela troca de informação, recursos e artefatos.

Ambientes Virtuais de Aprendizagem (AVA) integram Tecnologia da Informação e Comunicação (TIC), visando a criação de ambientes baseados na Internet que possibilitem o processo de construção do conhecimento e autonomia por parte de seus interagentes [Castro Filho et al. 2005]. Atualmente, educadores possuem acesso a tecnologias melhores e modernas [Gütl and Chang 2008]. Entretanto, apesar das tecnologias disponíveis, existe uma gama de fatores que influenciam no aproveitamento dos recursos, tais como: processos, estratégias, motivações e cultura.

O SOLAR (Sistema Online de Aprendizagem) é AVA cujo modelo de participação é orientado ao professor e ao aluno, possibilitando a publicação de cursos e interação com os mesmos. Atualmente possui versões web, mobile (Android e IOS) e MOOC (Massive Open Online Course). Adicionalmente tem-se o ECOS de e-learning SOLAR, com diversas integrações entre desenvolvimento, sociedade, pesquisa e negócios.

Para o ECOS SOLAR, diversas situações de evolução e manutenção fazem parte de seu cotidiano. Motivado pela falta de documentação ou atualização, mudanças de requisitos possuem impactos em todas as plataformas centrais do ECOS. Essas mudanças, sejam por defeitos identificados ou por customizações da plataforma para usuários ou para se ajustarem às plataformas de desenvolvimento, provocariam um efeito benéfico ao ambiente de desenvolvimento caso fossem modeladas, documentadas e analisadas quanto ao impacto de sua implementação em contextos diferentes.

No contexto de um ECOS, algumas questões surgem: (i) o que evoluir do SOLAR web e mobile; (ii) como modelar essas evoluções; e (iii) como modelar aspectos dinâmicos do SOLAR. O ECOS é estático do ponto de vista de evolução, pois havendo necessidade de uma nova funcionalidade, configuração ou personalização, a plataforma é alterada, testada e implantada de maneira estática, ou seja, não em tempo de execução. Assim, oportunidades e benefícios de mudanças dinâmicas se tornam atrativas para esse contexto.

Uma forma de modelar os aspectos dinâmicos é utilizar o modelo de features de uma Linha de Produto de Software Dinâmica (LPSD). Segundo Pascual et al. [Pascual et al. 2015], uma LPSD é um único sistema que é capaz de adaptar seu próprio comportamento em tempo de execução. O modelo de features representa as features do domínio e a variabilidade da linha. Já as features descrevem as funcionalidades e as características de qualidade de um produto de software [Benavides et al. 2010]. Uma maneira de representar os aspectos dinâmicos em um modelo de features é modelando as adaptações de contexto [Capilla et al. 2014]. Dessa forma, esse trabalho utilizará o modelo de features com contexto para modelar os aspectos dinâmicos do SOLAR.

A ideia deste trabalho é estudar variabilidade para se ter um software capaz de se adaptar às necessidades do usuário ou restrições de recursos. Esta adaptação é possível pelo adiamento da resolução de variabilidades para o tempo de execução. Neste caso, a resolução se dá no momento em que o sistema é inicializado ou durante sua execução.

O objetivo principal deste trabalho é discutir aspectos dinâmicos de modelagem de software do ECOS de e-learning SOLAR. Como objetivo secundário, pretende-se apre- 
sentar um exemplo focando na funcionalidade de fórum de discussão do AVA SOLAR, um dos serviços mais utilizados dentro do ECOS SOLAR. O artigo está dividido, além desta introdução, nas seguintes seções: uma breve discussão sobre o AVA SOLAR e o ECOS de e-learning SOLAR, um exemplo de modelagem dinâmica do fórum de discussão, análises e discussões, e por fim, conclusões e trabalhos futuros.

\section{Ambiente Virtual de Aprendizagem SOLAR}

O SOLAR é um AVA projetado para possibilitar a criação de um espaço virtual para cursos presenciais ou semi-presenciais, e baseia-se na utilização de software livre em uma arquitetura integrável a outros ambientes. Sua versão principal e mais completa é uma aplicação web. Atualmente ele conta com cerca de 47.000 usuários cadastrados, com média de 2.000 acessos diários. Ele serve como ponto de convergência para a criação do Blended Education, ou seja, a mescla de características de ambas as modalidades de Educação, para formação de um novo modelo educacional que utiliza fortemente as TICs.

$\mathrm{O}$ Blended Education ou Blended Learning é um derivado do e-learning, e referese a um sistema de formação onde a maior parte dos conteúdos é transmitido em curso à distância, normalmente pela internet. Porém, ele inclui necessariamente situações presenciais, daí a origem da designação "blended", algo misto, combinado. Ele pode ser estruturado com atividades síncronas, ou assíncronas, da mesma forma que o e-learning, ou seja, em situações onde professor e alunos trabalham juntos em horários pré-definidos, ou não, com cada um a cumprir suas tarefas em horários flexíveis.

O AVA SOLAR é composto pelos seguintes módulos: Núcleo de Integração de Módulos, Gerência de Usuários e Controle de Acesso, Integrador do AVA com o sistema de gestão acadêmica da universidade, Espaço Pessoal do Interagente, Avaliação e Acompanhamento, Ferramenta de Autoria para Conteúdos, Ferramentas de Comunicação, Ferramentas de Conteúdo, Ferramentas Administrativas, Ferramentas de Curso, e Ferramentas Colaborativas e Cooperativas. Algumas funcionalidades estão exibidas na Figura 1.

O AVA SOLAR é disponibilizado principalmente em sua versão web, sendo esta seu uso mais comum. Entretanto, duas versões web existem. Essas duas versões se diferem principalmente pela quantidade de novas funcionalidades adicionadas e pela interface gráfica que foi totalmente reformulada. A versão web mais antiga foi utilizada pela UAB, sendo atualmente usada apenas para recuperação de dados históricos de bases antigas. A Universidade Aberta do Brasil (UAB) ${ }^{1}$ é um sistema integrado por universidades

${ }^{1}$ Universidade Aberta do Brasil (UAB) - http://uab.capes.gov.br/
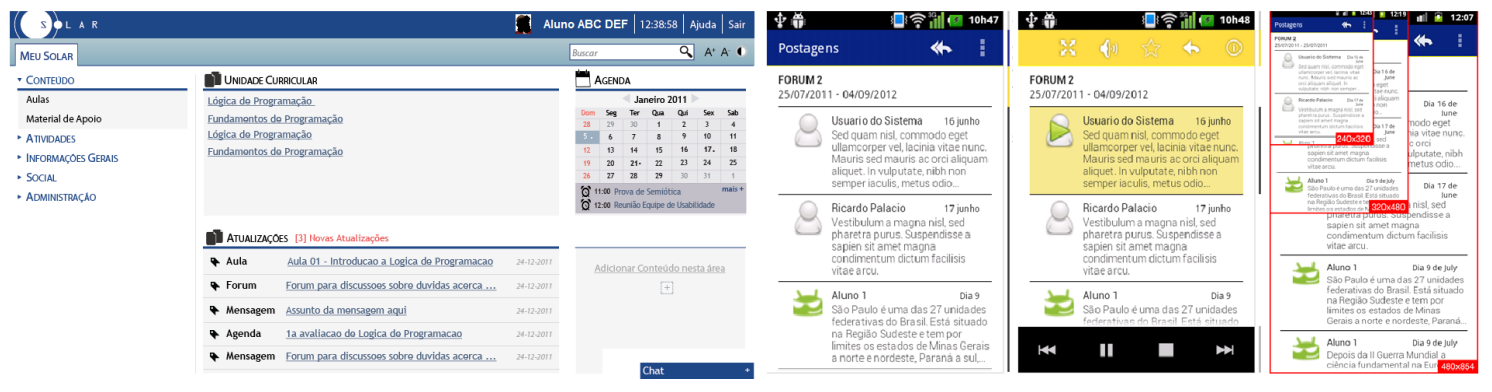

Figura 1. Exemplo de uma tela com funcionalidades do AVA SOLAR web e mobile 
públicas que oferece cursos de nível superior para a população com dificuldade de acesso à formação universitária, por meio da Educação à Distância (EAD). A versão web atual é considerada a versão oficial do SOLAR, usada atualmente em treinamentos, UAB e aulas de cursos presenciais. É a evolução em termos de tecnologia e usabilidade. Há também a versão mobile do SOLAR, que possui funcionalidades reduzidas, e também a versão MOOC (Curso Online Aberto e Massivo - Massive Open Online Course) do SOLAR.

\section{ECOS SOLAR}

Em torno do SOLAR, um conjunto de relacionamentos foi formado entre usuários, fornecedores de tecnologia, desenvolvedores de soluções e relações comerciais [Coutinho et al. 2017a]. Vários sistemas foram desenvolvidos em torno da plataforma central, e muitas versões e manutenção também surgiram. A disponibilização de uma API para a construção de soluções para a plataforma também contribuiu para a integração e difusão do ambiente. Nesse contexto, surgiu o ECOS SOLAR.

Uma SSN (Software Supply Network, rede de fornecimento de software) é uma série de software, hardware e serviços conectados que cooperam para atender às demandas do mercado [Jansen et al. 2007]. A Figura 2 apresenta uma modelagem em SSN para o ECOS SOLAR, baseada em [Boucharas et al. 2009] com a extensão proposta em [Costa et al. 2013]. O SOLAR e seus produtos são a Empresa de Interesse, apoiada por diversos tipos de fornecedores: software, hardware, equipes de desenvolvimento, bancos de dados e outros sistemas. Como intermediários, temos a equipe de desenvolvedores SOLAR, lojas de aplicativos e um comitê de pesquisa. Existem vários tipos de Clientes no ECOS SOLAR, desde instituições como universidades, usuários de plataformas, pesquisadores, sistemas de gerenciamento, até desenvolvedores externos, que por sua vez têm seus próprios clientes. A extensão da notação proposta propôs o papel do Agregador, que no caso do ECOS SOLAR é representado pelo Coordenador Técnico do SOLAR. Ele é responsável, além de gerenciar o desenvolvimento do SOLAR, de intermediar novos produtos e serviços, alinhados às necessidades do negócio, agregando valor e aproveitando as oportunidades que surgem com o uso da plataforma.

\section{Análise da Funcionalidade de Fórum de Discussão do AVA SOLAR}

O AVA SOLAR possui muitas funcionalidades. Para realizar uma análise de aspectos dinâmicos da plataforma, escolheu-se a funcionalidade do fórum de discussão. Essa escolha se deu por esta ser uma funcionalidade comum a qualquer AVA, e que possui um nível de utilização bastante elevado por seus usuários.

\subsection{Fórum de Discussão}

O fórum de discussão do AVA SOLAR é uma dos serviços mais utilizados por seus usuários, tanto na aplicação web quanto na mobile. Além de possuir características tradicionais de um fórum de discussão, tais como postar mensagens, responder mensagens, criar novos tópicos, organizar cronologicamente e anexar arquivos, ele possui algumas funcionalidades adicionais, como transcrição de texto para áudio e vice-versa. Alguns elementos de pesquisa e de demandas de usuários são identificados e podem ser adicionados, após um processo de desenvolvimento. Tais elementos também são desejáveis pelos usuários dos cursos à distância, como tutores e coordenadores de curso, e são específicos 


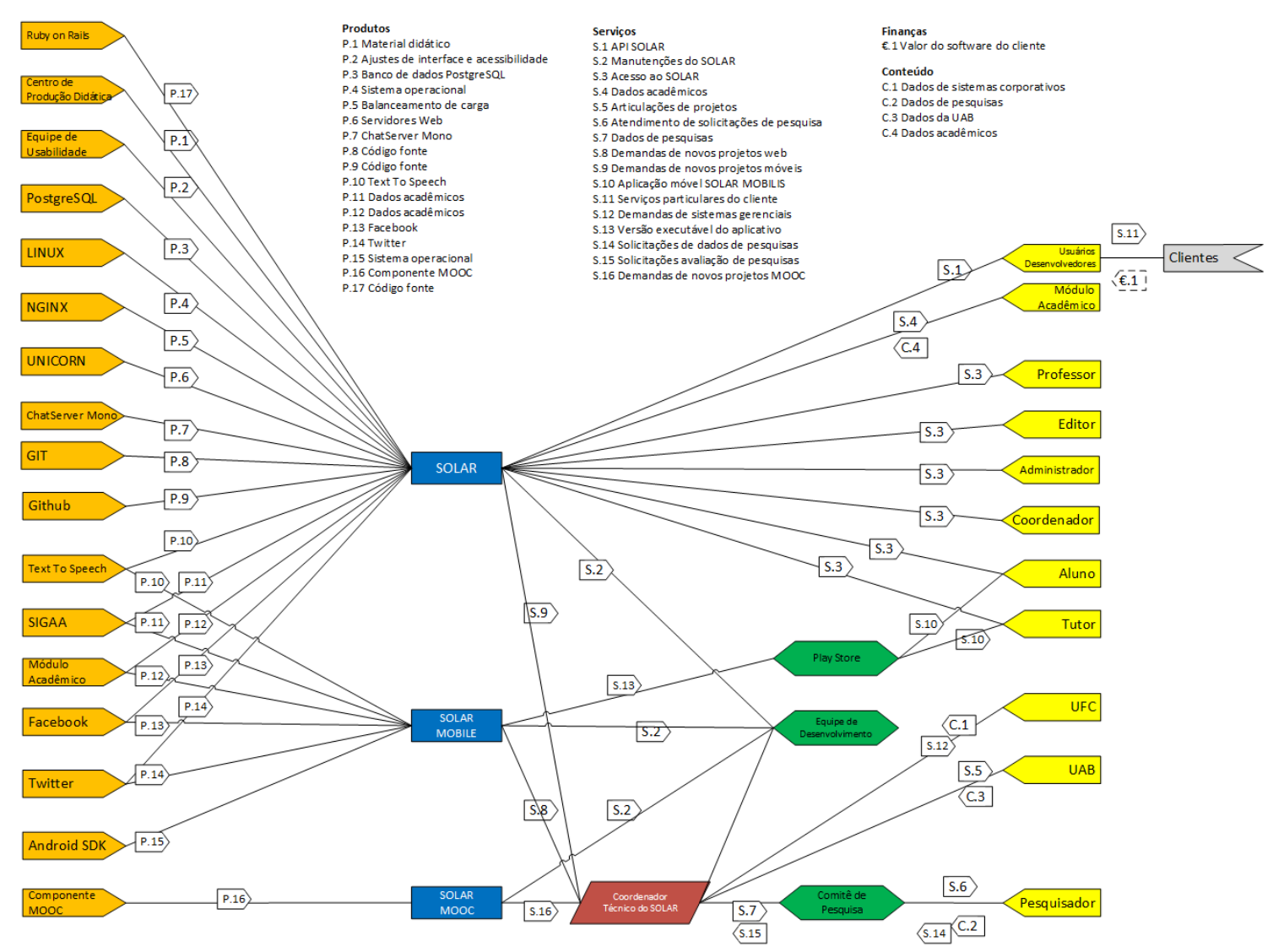

Figura 2. Modelagem SSN para o ECOS do AVA SOLAR [Coutinho et al. 2017a]

para o contexto da EAD. Essas funcionalidades seriam correção ou análise automática de fóruns de discussão, dicas e contabilização de métricas. Além disso, funcionalidades gerais, como alteração do idioma, também seriam interessantes de serem disponibilizadas.

Como impacto dessas funcionalidades para o AVA SOLAR e para os sistemas agregados (sistemas que utilizam dados do AVA SOLAR) tem-se uma melhoria do ambiente, agilidade em operações e processos, feedback maior e mais rápido para usuários e adequação às necessidades de clientes.

\subsection{Modelos de Features do Fórum de Discussão}

Embora os modelos de features sejam estudados na engenharia de domínio de uma Linha de Produto de Software, estes modelos de informação podem ser utilizados para outras áreas que vão desde a coleta dos requisitos, bem como a estrutura do modelo de dados. A estrutura de um modelo de features é representado como um conjunto hierarquizado de features composta por [Benavides et al. 2010]: (i) Relações entre uma feature pai e suas features filhas (ou subfeatures); e (ii) Restrições (ou cross-tree) que são tipicamente declarações de inclusão ou exclusão de features.

Existem várias ferramentas para modelagem do modelos de features, mas na literatura identificou-se apenas três ferramentas para modelagem de modelos de features com aspectos dinâmicos: MOSKitt4SPL [Alférez et al. 2014], VariaMos [Mazo et al. 2015] e DyMMer [Bezerra et al. 2016]. Somente a DyMMer estava disponível. Apenas foi identificado um repositório de modelos de features, o S.P.L.O.T. [Mendonca et al. 2009]. 
A ferramenta DyMMer (Dynamic feature Model tool based on Measures) foi desenvolvida para extrair medidas a partir de modelos de features, que podem ser descritas no formato do S.P.L.O.T., e suporta a edição de modelos de features e a extração automática de medidas de qualidade [Bezerra et al. 2016]. A DyMMer coleta um número grande de medidas de qualidade com a finalidade de apoiar a avaliação da manutenibilidade do modelo de features, 40 medidas no total, utiliza medidas específicas para modelos de features de Linhas de Produto de Software Dinâmicas e exporta, automaticamente, os valores das medidas para planilhas no formato Microsoft Excel.

A Figura 3(a) exibe o modelo de features na ferramenta S.P.L.O.T., e a Figura 3(b) exibe o modelo exportado para a ferramenta DyMMer [Bezerra et al. 2016]. As Figuras 4(a), (b) e (c) exibem configurações para contextos variados, modeladas na DyMMer.

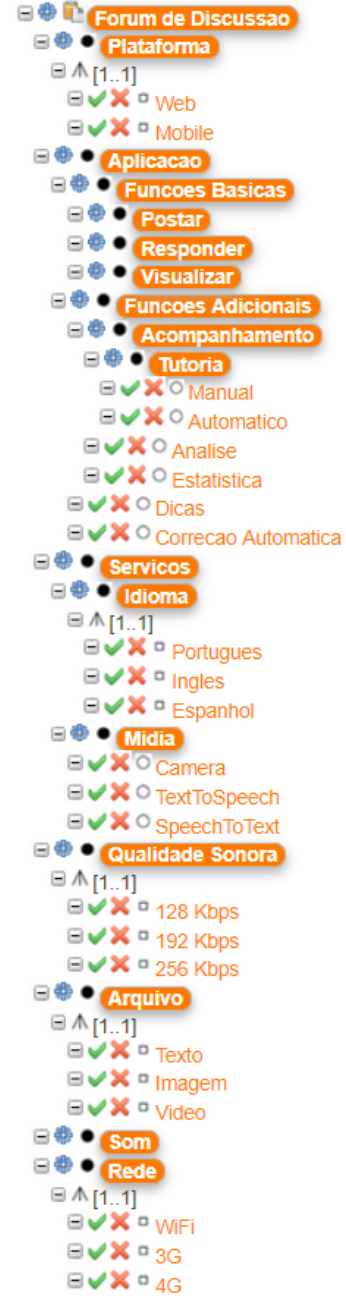

(a)

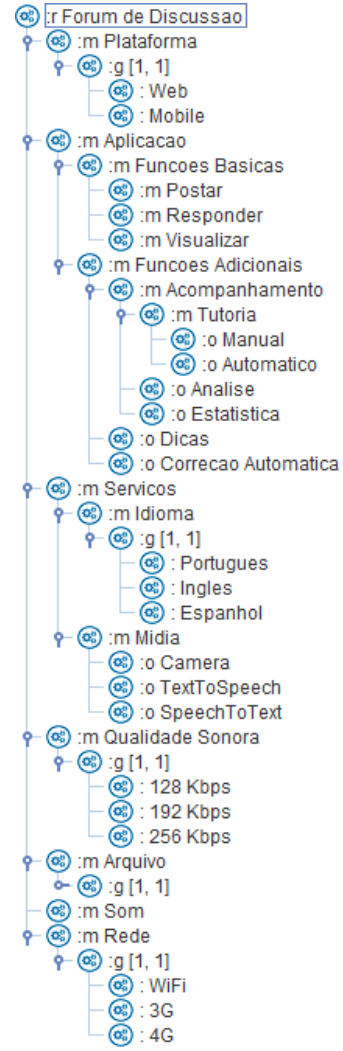

(b)

Figura 3. (a) Diagrama de features geral SPLOT [Mendonca et al. 2009] e (b) Diagrama de features geral DYMMER [Bezerra et al. 2016] 


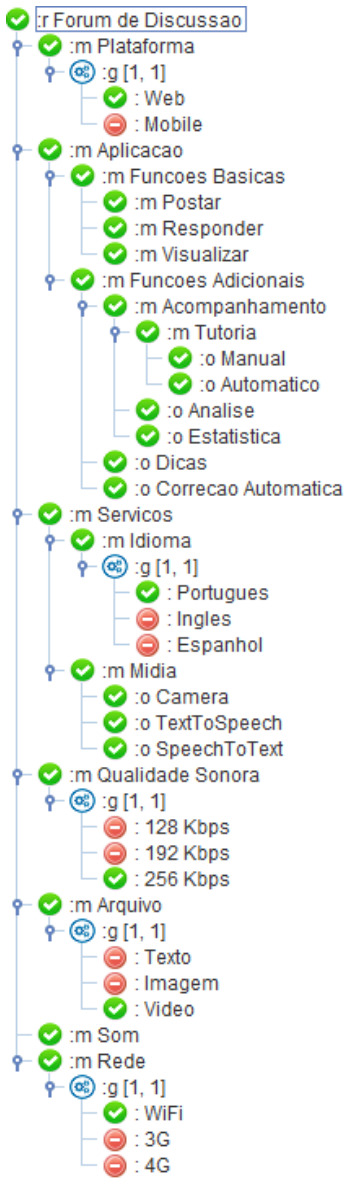

(a)

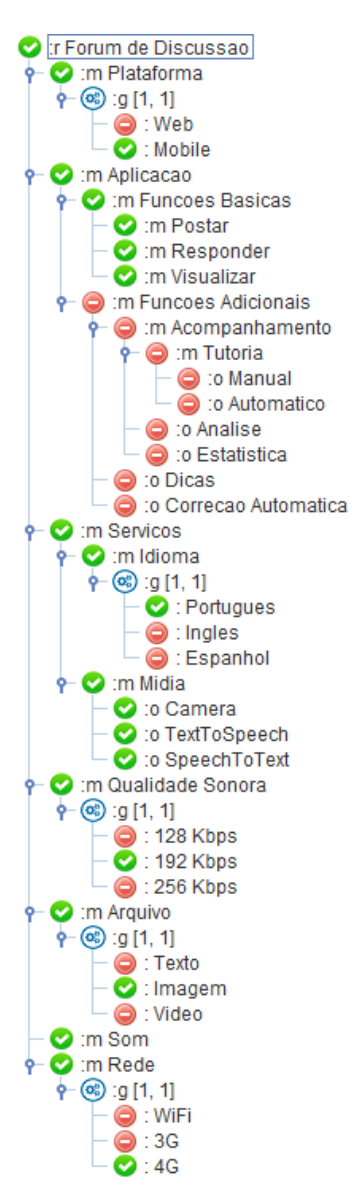

(b)

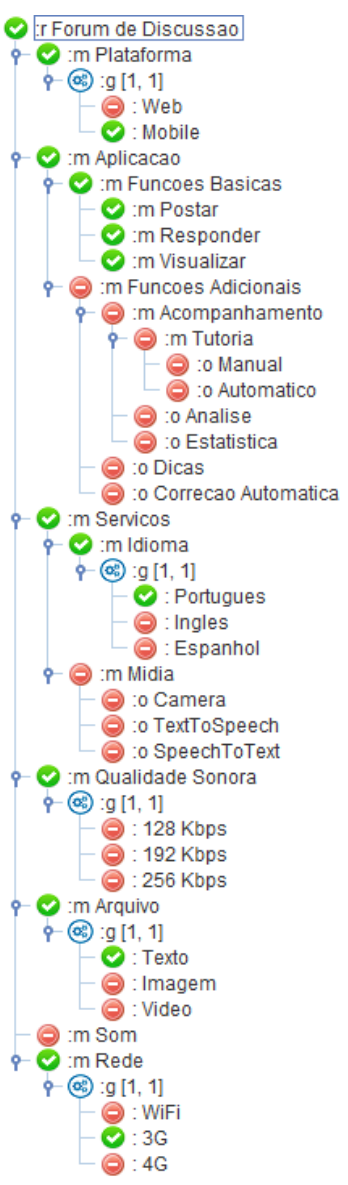

(c)

Figura 4. Diagramas de features modelados na ferramenta DYMMER com diferentes contextos: (a) Contexto 1 - acesso em ambiente com recursos, (b) Contexto 2 - acesso em ambiente móvel com conectividade boa e (c) Contexto 3 - acesso em ambiente móvel com conectividade sem qualidade

\subsubsection{Contextos}

Considere inicialmente um aluno de um curso à distância em sua residência, acessando o AVA SOLAR para aulas, conectado a seu notebook Nesse contexto, o aluno possui recursos altos de bateria e conectividade, não impactando na qualidade do serviço de áudio e vídeo. Este cenário está representado na Figura 4(a).

Em seguida, o aluno tem que ir para uma aula presencial, e continuará conectado ao AVA SOLAR em um veículo. Nesta situação, a bateria do notebook está mediana, e a conexão se dará por meio da rede de telefonia móvel, a qual consome mais recursos de energia. Nesse contexto, a qualidade sonora é reduzida, e recursos de vídeo não estarão mais disponíveis, apenas imagens. A Figura 4(b) está apresentando esta situação.

Por fim, a conexão com a telefonia móvel perde qualidade e a aplicação deve ser ajustada. Recursos de áudio e vídeo são desabilitados para economizar energia, e apenas é possível a utilização das funcionalidades básicas do fórum de discussão, com apenas texto sendo utilizado no fórum. Esta situação está retratada na Figura 4(c). 


\section{Análises e Discussões}

Sistemas de software intensivo são geralmente centrados em um plataforma de software de modo que uma atenção crescente tem sido dada para influenciar e para promover interdependência nas relações entre todas as partes envolvidas [Coutinho et al. 2017b]. A comunidade de Engenharia de Software tem utilizado a metáfora ecossistema para descrever os sistemas que geralmente são centralizados em uma plataforma de software. Nesse sentido, os dois conceitos se relacionam e promovem discussão e pesquisa.

O ECOS SOLAR possui um conjunto bem diversificado de desenvolvedores e usuários. As imagens da Figura 4 possibilitam visualizar apenas três contextos de um aspecto do ECOS SOLAR, relacionado a uma funcionalidade do AVA SOLAR, que é o fórum de discussão, sendo esta uma das mais utilizadas. Pela imagem é possível visualizar diversas tecnologias de software que são trabalhadas em diferentes momentos do ciclo de vida do desenvolvimento de software, desde requisitos, análise, implementação e testes.

Algumas métricas foram coletadas na ferramenta DyMMer [Bezerra et al. 2016], possibilitando algumas análises. O número de contextos do modelo de features é igual a 3, apenas porque modelou-se 3 contextos para essa análise. Diversos outros contextos poderiam ter sido modelados. O número de features ativadas, respectivamente para os três contextos, foram 31, 22 e 16. Percebe-se para a situação descrita como exemplo, que à medida em que os contextos ocorriam, a quantidade de fetaures ativadas ia diminuindo. Isso faz sentido, pois pela situação realmente os recursos foram ficando escassos, limitando sua utilização e adaptando a aplicação. Da mesma forma, o número de features desativadas foi aumentando (9, 18 e 24$)$.

As features de contexto são o número de features que estão sempre presentes no modelo de features, independentemente do contexto que está ativado, e para para os três cenários foram iguais a 12. Em geral são as funcionalidades que sempre devem existir para que a aplicação base seja executada plenamente.

O AFCA é o número de features ativadas em um contexto específico dividido pelo número de contextos. O DFCA é o número de features desativadas em um contexto específico dividido pelo número de contextos. AFCA resultou para os três cenários em 10.3, 7.3 e 5.3. DFCA resultou em 3, 6 e 8. Novamente, a medida em que as fetures foram sendo desativadas, AFCA diminuía, semelhante ao número de features ativadas. Da mesma forma ocorreu com DFCA e o número de features desativadas, sendo que estas aumentavam.

Respondendo às questões mencionadas na introdução, tem-se: (i) o que evoluir do SOLAR web e mobile: funcionalidades que podem ser muito úteis para a comunidade que utiliza o SOLAR são as de acompanhamento, principalmente se automatizadas; (ii) como modelar essas evoluções: a documentação e modelagem de funcionalidades de maneira mais tradicional não é fácil de se manter no ambiente de desenvolvimento do SOLAR. Estratégias de modelagem ágil ou DevOPS seriam muito bem vindas para acelerar o processo de desenvolvimento e pô-las em prática; (iii) como modelar aspectos dinâmicos do SOLAR: algumas funcionalidades do SOLAR, como acompanhamento e customização de características conforme a plataforma, são aspectos que possuem uma dinamicidade atraente para o SOLAR, principalmente em contextos variados. Tais funções poderiam ser configuradas para se adequarem às necessidades de cada usuário do SOLAR. Mode- 
los que representassem a dinâmica do sistema em tempo real também seriam bastante úteis. Entretanto, ainda é um desafio como modelar aspectos dinâmicos do sistema e implementá-los.

\section{Conclusões}

Se considerarmos um sistema intensivo em software como aqueles em que software é um elemento dominante e essencial, tanto em sua estrutura quanto como um elemento transversal às etapas de produção, impactando substancialmente no planejamento, desenvolvimento e evolução desses sistemas, temos o AVA SOLAR encaixado-se nessa categoria. Seu desenvolvimento em suas diversas modalidades (web, mobile e MOOC) permeia diversos níveis de desenvolvimento, instituições demandantes, usuários pessoais e institucionais, parcerias e oportunidades de negócio. Sua evolução acompanha necessidades de diversos cursos online e alinhamento com tecnologias de suporte, como serviços para acessibilidade e laboratórios virtuais.

Este trabalho discutiu aspectos de variabilidade dinâmica com exemplo da funcionalidade fórum de discussão, modelado por meio de um diagrama de features. Percebeuse que a complexidade de se modelar aspectos dinâmicos e variabilidade é uma tarefa complexa, e requer muito esforço. Como trabalhos futuros pretende-se aprofundar na modelagem de outros aspectos dinâmicos do ECOS SOLAR, com diferentes modelos.

\section{Agradecimentos}

Este trabalho foi parcialmente suportado com recursos dos seguintes projetos: CrOSSiNg: Avaliação da Qualidade de Nuvens Computacionais Apoiadas por Redes Definidas por Software e Virtualização de Funções de Rede, Edital Universal MCTI/CNPq 01/2016 (processo 422342/2016-5).

\section{Referências}

Alférez, G. H., Pelechano, V., Mazo, R., Salinesi, C., and Diaz, D. (2014). Dynamic adaptation of service compositions with variability models. Journal of Systems and Software, 91:24-47.

Benavides, D., Segura, S., and Ruiz-Cortés, A. (2010). Automated analysis of feature models 20 years later: A literature review. Information Systems, 35(6):615 - 636.

Bezerra, C. I., Barbosa, J., Freires, J. H., Andrade, R. M. C., and Monteiro, J. M. (2016). Dymmer: a measurement-based tool to support quality evaluation of dspl feature models. In Proceedings of the 20th International Systems and Software Product Line Conference, pages 314-317. ACM.

Boucharas, V., Jansen, S., and Brinkkemper, S. (2009). Formalizing software ecosystem modeling. In Proceedings of the 1st International Workshop on Open Component Ecosystems, IWOCE '09, pages 41-50, New York, NY, USA. ACM.

Capilla, R., Ortiz, , and Hinchey, M. (2014). Context variability for context-aware systems. Computer, 47(2):85-87.

Castro Filho, J. A., Loureiro, R. C., Paula, P. S., Sarmento, W. W. F., Peixoto, L. E., Pequeno, H. S. L., Rocha, B. T. S., and Viana Júnior., G. S. (2005). Portal humanas: Um ambiente colaborativo para criação de projetos e comunidades virtuais para a área de humanidades. In XVI Simpósio Brasileiro de Informática na Educação (SBIE 2005). 
Costa, G., Silva, F., Santos, R., Werner, C., and Oliveira, T. (2013). From applications to a software ecosystem platform: An exploratory study. In Proceedings of the Fifth International Conference on Management of Emergent Digital EcoSystems, MEDES '13, pages 9-16, New York, NY, USA. ACM.

Coutinho, E. F., Santos, I., and Bezerra, C. I. M. (2017a). A software ecosystem for a virtual learning environment: Solar seco. In 2017 IEEE/ACM Joint 5th International Workshop on Software Engineering for Systems-of-Systems and 11th Workshop on Distributed Software Development, Software Ecosystems and Systems-of-Systems (JSOS), pages 41-47.

Coutinho, E. F., Viana, D., and d. Santos, R. P. (2017b). An exploratory study on the need for modeling software ecosystems: The case of solar seco. In 2017 IEEE/ACM 9th International Workshop on Modelling in Software Engineering (MiSE), pages 47-53.

Gütl, C. and Chang, V. (2008). The use of web 2.0 technologies and services to support elearning ecosystem to develop more effective learning environments. In In proceedings of ICDEM 2008, pages 145-148.

ISO (2011). Iso/iec standard for systems and software engineering - systems and software engineering - architecture description - iso/iec 42010 ieee:2011.

Jansen, S., Brinkkemper, S., and Finkelstein, A. (2007). Providing Transparency in the Business of Software: A Modeling Technique for Software Supply Networks, pages 677-686. Springer US, Boston, MA.

Jansen, S., Brinkkemper, S., and Finkelstein, A. (2009). Business network management as a survival strategy: A tale of two software ecosystems. In Proceedings of the First International Workshop on Software Ecosystems, 11th International Conference on Software Reuse, pages 34-48.

Mazo, R., Muñoz-Fernández, J. C., Rincón, L., Salinesi, C., and Tamura, G. (2015). Variamos: an extensible tool for engineering (dynamic) product lines. In Proceedings of the 19th International Conference on Software Product Line, pages 374-379. ACM.

Mendonca, M., Branco, M., and Cowan, D. (2009). S.p.l.o.t.: Software product lines online tools. In Proceedings of the 24th ACM SIGPLAN Conference Companion on Object Oriented Programming Systems Languages and Applications, OOPSLA '09, pages 761-762, New York, NY, USA. ACM.

Messerschmitt, D. and Szyperski, C. (2003). Software Ecosystem: Understanding an Indispensable Technology and Industry. The MIT Press, 1 edition.

Oquendo, F. (2016). Formally describing the architectural behavior of software-intensive systems-of-systems with sosadl. In 201621 st International Conference on Engineering of Complex Computer Systems (ICECCS), pages 13-22.

Pascual, G. G., Lopez-Herrejon, R. E., Pinto, M., Fuentes, L., and Egyed, A. (2015). Applying multiobjective evolutionary algorithms to dynamic software product lines for reconfiguring mobile applications. Journal of Systems and Software, 103:392-411. 